\title{
Metabolism of Antarctic micronektonic Crustacea as a function of depth of occurrence and season
}

\author{
J. J. Torres ${ }^{1}$, A.V. Aarset ${ }^{2}$, J. Donnelly ${ }^{1}$, T. L. Hopkins ${ }^{1}$, T. M. Lancraft ${ }^{1}$, D. G. Ainley ${ }^{3}$ \\ ${ }^{1}$ Dept of Marine Science, University of South Florida, 140 Seventh Ave. South, St. Petersburg, Florida 33701, USA \\ ${ }^{2}$ Lilleveien 5, N-6006 Ålesund, Norway \\ ${ }^{3}$ Point Reyes Bird Observatory, 4990 Shoreline Hwy, Stinson Beach, California 94970, USA
}

\begin{abstract}
Oxygen consumption rates were determined on 21 species of crustaceans typical of the Southern Ocean micronektonic crustacean assemblage during spring (November), fall (March), and winter (June-August). Specimens were collected in the Scotia-Weddell Sea region in the vicinity of $60^{\circ} \mathrm{S}, 40^{\circ} \mathrm{W}$ in the upper $1000 \mathrm{~m}$ of the water column. Respiration $\left(y, \mu l \mathrm{O}_{2} \mathrm{mg}^{-1}\right.$ wet mass $\left.\mathrm{h}^{-1}\right)$ declined with depth of occurrence $(x, m)$ according to the equation $y=0.125 x^{-0.172 \pm 0052}(p<0.05)$ despite the isothermal character of the water column, suggesting that lower metabolic rates are a temperatureindependent adaptation to life in the deep sea. Three species of Crustacea showed a lowered metabolism during the winter season: the krill Euphausia superba and the 2 hyperiid amphipods Cyllopus lucasii and Vibilia stebbingi. Critical oxygen partial pressure ( $\mathrm{PC}$ ) varied between 29 and $52 \mathrm{~mm} \mathrm{Hg}$, well below the lowest $\mathrm{PO}_{2}$ found in the water column. It is suggested that the long nights of the Antarctic winter decrease the effectiveness of visual predation in the epipelagic zone, allowing lowered metabolic rates to be a viable overwintering strategy for some species.
\end{abstract}

KEY WORDS: Metabolism - Antarctic · Pelagic Crustacea $\cdot$ Overwinter

\section{INTRODUCTION}

The micronektonic crustacean fauna of the Southern Ocean is comprised of 2 distinct faunal groups: representatives of a cosmopolitan pelagic fauna (mainly deep living) and species which are found only in waters south of the Antarctic convergence (Nagata 1986, Iwasaki \& Nemoto 1987, Lancraft et al. 1989, 1991). Virtually all of the groups considered to be classical midwater crustaceans, including the deepliving mysids and ostracods, are absent south of the Weddell-Scotia confluence, which coincidentally is the northward limit of winter sea ice in the Atlantic sector. The overall result of the faunal changes is that the character of a midwater trawl catch in the Antarctic is vastly different from that of other regions of the world ocean.

Three characteristics of the Antarctic pelagial are unique to the system. First, temperatures are uniformly cold in the horizontal and vertical planes. Sea surface temperatures vary between -1.87 and $0^{\circ} \mathrm{C}$ near the continent to -1 to $4^{\circ} \mathrm{C}$ near the convergence (Gordon et al. 1982). The remainder of the water column is nearly isothermal with temperatures changing less than $2^{\circ} \mathrm{C}$ between 100 and $1000 \mathrm{~m}$. Second, sea ice cover expands and contracts with the seasons, altering the light field of the water column beneath it. In the Atlantic sector, winter sea ice extends to the WeddellScotia confluence, located at a latitude of approximately $60^{\circ} \mathrm{S}$, thus covering all of the Weddell Sea and part of the southern Scotia Sea at its maximum extent (Zwally et al. 1983). Third, day length varies with latitude from 0 to $6 \mathrm{~h}$ in winter to 18 to $24 \mathrm{~h}$ in summer (Smith \& Sakshaug 1990). As a consequence, primary production varies from near zero in the winter months to local maxima of greater than $500 \mathrm{mg} \mathrm{C} \mathrm{m}^{-2} \mathrm{~d}^{-1}$ at the spring ice edge (Smith \& Nelson 1990).

Pronounced seasonal changes in the physical and biological environment of the Southern Ocean alter the character of the resource spectrum available to the micronektonic Crustacea. Not only is winter primary production near zero (W. O. Smith unpubl. data), pat- 
terns of zooplankton distribution are quite different in the winter months; zooplankton biomass is reduced in the upper $100 \mathrm{~m}$ of the water column (Hopkins et al 1993) with at least 2 of the dominant species of copepods descending to mesopelagic depths to overwinter in a diapause state (Hopkins et al. 1993). The seasonal changes in available food energy have a potentially profound influence on both feeding success and metabolic processes. The nature of that influence will vary with the trophic position of the species. For example, primarily herbivorous species such as Euphausia superba face several months of low algae production and therefore low food during the winter season. In contrast, deeper-living carnivorous species such as Pasiphaea scotiae might be expected to capitalize on the populations of diapausing copepods at mesopelagic depths as a lipid-rich and non-motile food source (Alldredge et al. 1984, Everson 1984). It was the purpose of this study to describe the metabolic rates of a broad range of Southern Ocean micronektonic crustacean species of various taxa and depth distributions. The study was designed to provide basic information on metabolism of Antarctic species for comparison with those living in other ocean systems and to examine the influence of season on metabolic rate.

\section{MATERIALS AND METHODS}

Collection of specimens. Crustaceans were collected using either mouth-closing Tucker trawls (9.0 $\mathrm{m}^{2}$ or $2.25 \mathrm{~m}^{2}$ mouth area) or downward-looking, vertically deployed plummet nets $\left(1 \mathrm{~m}^{2}\right.$ mouth area). Tucker trawls were equipped with either blind or thermal-turbulence-protecting cod-ends (Childress et al. 1978); plummet nets terminated in blind cod-ends only. Specimens were taken in the upper $1000 \mathrm{~m}$ of the water column in the vicinity of the marginal ice zone during spring (November-December) 1983, fall (March) 1986, and winter (June-August) 1988 as part of the AMERIEZ (Antarctic Marine Ecosystem Research at the Ice Edge Zone) program to study ice edge biology. Sampling locations were all in the Scotia-Weddell Sea region but moved with seasonal movement of the pack ice edge. Thus, spring and winter collections were in the Scotia Sea in the vicinity of $60^{\circ} \mathrm{S}, 40^{\circ} \mathrm{W}$; fall sampling took place further south, $65^{\circ} \mathrm{S}, 46^{\circ} \mathrm{W}$. Collections were made on a continuum from deep in the pack ice out to $300 \mathrm{~km}$ seaward of the ice edge in fall and winter In spring, collections were made in the open water only. Station locations are given in Donnelly et al. (1990).

The entire catch from each deployment of the Tucker trawl was transferred into seawater at approximately $0^{\circ} \mathrm{C}$ immediately on reaching the deck. Plummet net catches were transferred as quickly as possible, typically within 30 min. Individuals were removed for respiratory measurements and placed in 281 cylindrical tanks at a temperature of 0 to $1^{\circ} \mathrm{C}$, where they were held for not more than $3 \mathrm{~h}$ before being placed in respirometers. A $3 \mathrm{~h}$ holding time was chosen as the best compromise between allowing the individuals to recover from the stress of capture and minimizing any effects of prolonged starvation. All specimens used in respiratory measurements were in excellent condition: very lively and with no visible damage. Specimens in equivalent condition could be maintained indefinitely in the laboratory,

Respiratory measurements. Oxygen consumption rates were determined by allowing individuals to deplete the oxygen in a sealed container filled with filtered $(0.45 \mu \mathrm{m}$ pore size) seawater. Temperature was maintained at $0.5^{\circ} \mathrm{C}\left(+0.1^{\circ} \mathrm{C}\right)$ using a refrigeraled water bath. Oxygen partial pressure $\left(\mathrm{PO}_{2}\right)$ was continuously monitored using a Clark polarographic oxygen electrode (Clark 1956) as an individual reduced oxygen levels to low (10 to $20 \mathrm{~mm} \mathrm{Hg}$ ) partial pressures. Electrodes were calibrated using air- and nitrogen-saturated seawater at the experimental temperature. The time required for consumption of oxygen to low levels varied from 12 to $18 \mathrm{~h}$. Streptomycin and Neomycin (each $25 \mathrm{mg} \mathrm{l}^{-1}$ ) were added to the seawater to minimize microbial growth. To control for possible oxygen consumption by microorganisms, an individual was removed after selected runs, its volume was replaced with fresh seawater, and oxygen consumption was again measured for 2 to $4 \mathrm{~h}$. In all cases microbial oxygen consumption was negligibly low.

Water-jacketed, rectangular, lucite chambers were used for intermediate-sized and larger (wet mass, WM $>50 \mathrm{mg}$ ) individuals. In this design, a perforated lucite false bottom isolated the experimental subject from a stir bar; stir bars rotated at the minimum speed needed to assure proper function of the oxygen electrodes. Chamber sizes used for intermediate and larger-sized individuals varied from 50 to $1350 \mathrm{ml}$ depending on individual size. For example, intermediate-sized krill $(45 \mathrm{~mm}$ ) were placed in chambers 200 to $250 \mathrm{ml}$ in volume. Individuals were allowed to swim freely within the confines of a chamber. Most often, after a brief period of high activity immediately following introduction into the chamber, individuals settled into a low-level sculling with the pleopods to maintain station at mid-depth in the chamber.

Smaller Iucite chambers or plastic syringes were used to accomodate the smallest individuals. Microcathode oxygen electrodes were machined to act as plungers in the barrels of $10 \mathrm{ml}$ plastic syringes which 
had had their ends cut off. The respiratory chamber within the syringe barrel was defined at one end by the syringe plunger and at the other by the oxygen electrode. Microcathode oxygen electrodes require no stirring; they were used in both the small lucite and syringe barrel respirometers. All experiments took place in the dark with brief periods of observation in low light.

Data were recorded using a computer-controlled digital data-logging system. Each oxygen probe was scanned once per minute, its signal averaged over a period of $1 \mathrm{~s}$, and then recorded. Data were reduced by first averaging the 30 recorded values in each $30 \mathrm{~min}$ increment of an entire 12 to $18 \mathrm{~h}$ experiment, producing 24 to 3630 -min points per run. Data obtained during the first hour were discarded due to the high activity of individuals just after introduction to the respirometer. All 30-min points thereafter, down to an oxygen partial pressure of about $60 \mathrm{~mm} \mathrm{Hg}$, were averaged to produce a routine rate for each individual. Maximum rates were the maximum 30 -min rate and minimum rates the minimum 30-min rate recorded for each individual after the first hour, but above a $\mathrm{PO}_{2}$ of $60 \mathrm{~mm} \mathrm{Hg}$. Maximum rates were usually associated with the beginning of an experiment, minimum rates were most commonly recorded during the middle of a run. Critical oxygen partial pressures $\left(\mathrm{Pc}_{\mathrm{C}}\right.$ ) were assigned at the $\mathrm{PO}_{2}$ where the oxygen consumption rate declined precipitously toward zero.

Data. Mass variability within many species was sufficiently large to necessitate breaking up the data into size classes (cf. Ikeda 1988). Size classes were arbitrarily created by halving each order of magnitude change in mass, e.g. size class 1: 0.0000 to $0.0049 \mathrm{~g}$, size class 2: 0.0050 to $0.0099 \mathrm{~g}$, size class 3: 0.010 to $0.049 \mathrm{~g}$, size class 4: 0.050 to $0.099 \mathrm{~g}$, etc. Size classes are best illustrated in the data on Euphausia superba in Table 3.

Individual runs were grouped according to season and size class and tested for differences using either ANOVA or, if variances were not homogeneous, a Kruskal-Wallis test. Interspecific comparisons were done by using the data from the largest individual size class of each species in the spring or fall seasons. If there were no significant differences, the data for the 2 seasons were lumped to produce a common mean. In 5 cases winter data were used due to a lack of data for the other 2 seasons. Species for which total $n<3$ were not used in statistical analyses; they are presented in Tables 1 to 4 for purposes of comparison only. Analyses were done using the summary data presented in Table 5. Regressions were done using the leastsquares method. Analysis of covariance was performed using the methods outlined in Snedecor \& Cochran (1967).
Mass-normalized respiratory data ( $a$ in Table 5) were calculated using a $b$-value of 0.75 to standardize to a mass of $1 \mathrm{~g}$. Data were temperature corrected using a $\mathrm{Q}_{10}$ of 2.0. Minimum depths of occurrence (MDO, that depth below which about $90 \%$ of the population lives; Childress \& Nygaard 1973) for the species examined by lkeda (1988) were taken from distribution data in Tables 1 to 4

Depth distributions. Vertical distributions were taken directly from Lancraft et al. $(1989,1991)$ or from unpublished data acquired on the species from the hundreds of opening-closing net tows executed during the AMERIEZ trawling program. Depths shown in Tables 1 to 5 are considered for purposes of analysis to be equivalent to minimum depth of occurrence. They are in fact the shallowest depth of capture for the species. Seasonal differences in vertical distributions were observed and these are reflected in the values reported in Tables 1 to 5 .

\section{RESULTS}

\section{Grammarid amphipods}

Routine oxygen consumption rates varied from $0.015 \mu \mathrm{O}_{2} \mathrm{mg}^{-1} \mathrm{WM} \mathrm{h}^{-1}$ in the large bathypelagic amphipod Euandania gigantea to $0.137 \mathrm{\mu l} \mathrm{O}_{2} \mathrm{mg}^{-1}$ $\mathrm{WM} \mathrm{h}^{-1}$ in the under-ice (sympagic) species Eusirus antarcticus (Table 1). Maximum rates were consistently about double the routine rates $(\bar{x} \pm \mathrm{SD}, 2.07 \pm$ 0.82). Maximum to minimum ratios were more highly variable with an overall mean of $6.64( \pm 5.91)$. Values for PC were obtained for the 2 species of Cyphocaris, 33 and $31 \mathrm{~mm} \mathrm{Hg} \mathrm{O}_{2}$ for $C$. faueri and $C$. richardi respectively. Eusirus antarcticus showed a slightly higher value: $40 \mathrm{~mm} \mathrm{Hg} \mathrm{O}$. The Pc-values exhibited by these 3 species are well below the minimum oxygen partial pressure found within their vertical range and within the Scotia-Weddell Sea region in general, ca $100 \mathrm{~mm} \mathrm{Hg}$ (Gordon et al. 1982), suggesting that oxygen is not a limiting variable within the water column.

Data were available in 2 seasons for 3 species (Cyphocaris faueri, C. richardi, and Parandania boecki) but only in $P$. boecki were there sufficient numbers in any one size class to attempt a seasonal comparison. No significant effect of season on routine metabolism was observed ( $p>0.05$, ANOVA).

Sufficient numbers and size differences were available in the routine rates of Cyphocaris richardi to test for effects of mass on metabolism. The relation was described by the equation $y=0.068 x^{1.05 \pm 0.072}(b \pm \mathrm{SE}$, $r^{2}=0.968, p>0.05$ ), suggesting that metabolism scales directly with mass in this species. 
Table 1. Oxygen consumption rates of gammarid amphipods. All values expressed as mean (SD). To use conversion factors multiply wet-mass-specific rate by indicated value to yield dry-mass-specific or ash-free dry-mass-specific rates. Size classes and depth distributions determined as described in text. $\mathrm{n}$ : number of replicates used in determining routine rates. MDO: minimum depth of occurrence; Pc: critical oxygen partial pressure; WM: wet mass; DM: dry mass; AFDM: ash-free dry-mass. F: falli S: spring; W: winter

\begin{tabular}{|c|c|c|c|c|c|c|c|c|c|c|}
\hline \multirow[t]{2}{*}{ Species $(\mathrm{n})$} & \multirow[t]{2}{*}{ Season } & \multirow{2}{*}{$\begin{array}{l}\mathrm{MDO} \\
(\mathrm{m})\end{array}$} & \multirow{2}{*}{$\begin{array}{c}\text { Mean } \\
\text { wet mass } \\
(\mathrm{g})\end{array}$} & \multirow{2}{*}{$\begin{array}{l}\text { Size } \\
\text { class }\end{array}$} & \multirow{2}{*}{\multicolumn{3}{|c|}{$\begin{array}{l}\text { Oxygen consumption rate } \\
\quad\left(\mu \mathrm{Ol} \mathrm{O}_{2} \mathrm{mg}^{-1} \mathrm{WM} \mathrm{h}^{-1}\right)\end{array}$}} & \multirow{2}{*}{$\begin{array}{c}\mathrm{Pc} \\
(\mathrm{mm} \mathrm{Hg})\end{array}$} & \multicolumn{2}{|c|}{ Conversions } \\
\hline & & & & & & & & & $\mathrm{DM}$ & AFDM \\
\hline Cyphocaris faueri (1) & $\mathrm{F}$ & 40 & 0.352 & 5 & 0.108 & 0.049 & 0.231 & & 4.24 & 5.42 \\
\hline C. faueri (6) & $\mathrm{S}$ & 40 & $\begin{array}{c}1.275 \\
(0.427)\end{array}$ & 7 & $\begin{array}{c}0.053 \\
(0.024)\end{array}$ & $\begin{array}{c}0.039 \\
(0.004)\end{array}$ & $\begin{array}{c}0.067 \\
(0.003)\end{array}$ & 33 & 4.24 & 5.42 \\
\hline C. faueri (2) & $\mathrm{F}$ & 40 & $\begin{array}{c}1.147 \\
(0.286)\end{array}$ & 7 & $\begin{array}{c}0.063 \\
(0.020)\end{array}$ & $\begin{array}{c}0.020 \\
(0.000)\end{array}$ & $\begin{array}{c}0.135 \\
(0.001)\end{array}$ & & 4.24 & 5.42 \\
\hline C. faueri (1) & W & 1000 & 0.865 & 6 & 0.037 & 0.016 & 0.079 & & 4.24 & 5.42 \\
\hline C. richardi (1) & $\mathrm{s}$ & 340 & 0.072 & 4 & 0.126 & 0.011 & 0.354 & 31 & 3.97 & 5.18 \\
\hline C. richardi (2) & W & 100 & $\begin{array}{c}0.066 \\
(0.040)\end{array}$ & 4 & $\begin{array}{c}0.073 \\
(0.006)\end{array}$ & $\begin{array}{c}0.019 \\
(0.011)\end{array}$ & $\begin{array}{c}0.164 \\
(0.089)\end{array}$ & 31 & 3.97 & 4.82 \\
\hline C. richardi (3) & $\mathrm{s}$ & 340 & $\begin{array}{c}0.376 \\
(0.078)\end{array}$ & 5 & $\begin{array}{c}0.084 \\
(0.014)\end{array}$ & $\begin{array}{c}0.038 \\
(0.016)\end{array}$ & $\begin{array}{c}0.131 \\
(0.018)\end{array}$ & 31 & 3.97 & 4.82 \\
\hline C. richardi (2) & $\mathrm{F}$ & 340 & $\begin{array}{c}0.698 \\
(0.067)\end{array}$ & 6 & $\begin{array}{c}0.107 \\
(0.012)\end{array}$ & $\begin{array}{c}0.034 \\
(0.024)\end{array}$ & $\begin{array}{c}0.185 \\
(0.012)\end{array}$ & & 3.97 & 4.82 \\
\hline Euandania gigantea (1) & $\mathrm{S}$ & 1000 & 5.813 & 8 & 0.015 & 0.008 & 0.029 & & 19.17 & 21.01 \\
\hline Eusirus antarcticus (26) & $\mathrm{F}$ & 0 & $\begin{array}{c}0.047 \\
(0.009)\end{array}$ & 3 & $\begin{array}{c}0.137 \\
(0.027)\end{array}$ & $\begin{array}{c}0.054 \\
(0.026)\end{array}$ & $\begin{array}{c}0.279 \\
(0.084)\end{array}$ & 42 & 2.56 & 3.03 \\
\hline E. microps (1) & $\mathrm{F}$ & 0 & 0.699 & 6 & 0.083 & 0.009 & 0.179 & & 3.07 & 3.60 \\
\hline E. propeperdentatus (2) & $\mathrm{F}$ & 0 & $\begin{array}{c}0.073 \\
(0.003)\end{array}$ & 4 & $\begin{array}{c}0.082 \\
(0.010)\end{array}$ & $\begin{array}{c}0.038 \\
(0.003)\end{array}$ & $\begin{array}{c}0.176 \\
(0.023)\end{array}$ & & 3.60 & 4.50 \\
\hline Parandania boecku (1) & W & 500 & 0.071 & 4 & 0.076 & 0.009 & 0.230 & & 6.51 & 9.02 \\
\hline P. boecki (6) & $\mathrm{S}$ & 500 & $\begin{array}{c}0.465 \\
(0.117)\end{array}$ & 5 & $\begin{array}{c}0.041 \\
(0.016)\end{array}$ & $\begin{array}{c}0.023 \\
(0.018)\end{array}$ & $\begin{array}{c}0.061 \\
(0.021)\end{array}$ & & 6.13 & 8.64 \\
\hline P. boecki (3) & w & 500 & $\begin{array}{c}0.425 \\
(0.120)\end{array}$ & 5 & $\begin{array}{l}0.037 \\
(0.007)\end{array}$ & $\begin{array}{c}0.007 \\
(0.003)\end{array}$ & $\begin{array}{c}0.150 \\
(0.111)\end{array}$ & & 6.13 & 8.64 \\
\hline
\end{tabular}

As a group, the gammarids are well represented throughout the water column, showing minimum depths of occurrence from 0 to $1000 \mathrm{~m}$. The eusirids predominate in the upper $100 \mathrm{~m}$, with the cyphocarids dominating from 100 to $500 \mathrm{~m}$. Below $500 \mathrm{~m}$ Parandania boecki is the most common gammarid, with Euandania gigantea being present, but very rare, below $1000 \mathrm{~m}$. P. boecki and E. gigantea, the 2 deepest-dwelling species, show the lowest routine rates (Table 1). The routine rates of species living above $\left(\bar{x}=0.090 \mu \mathrm{O}_{2} \mathrm{mg}^{-1} \mathrm{WM} \mathrm{h}^{-1}\right)$ and below $\left(0.028 \mathrm{\mu l} \mathrm{O}_{2} \mathrm{mg}^{-1} \mathrm{WM} \mathrm{h}{ }^{-1}\right) 500 \mathrm{~m}$ are significantly different (Kruskal-Wallis, $\mathrm{p}<0.05$ ). If effects of mass are minimized by normalizing all rates to a mass of $1 \mathrm{~g}$ (see Table 5), the rates above and below $500 \mathrm{~m}$ remain significantly different (Kruskal-Wallis, $p<0.05)$. Rates expressed as dry or ash-free dry mass show no trend with depth.

\section{Hyperiid amphipods}

Routine rates varied from $0.051 \mu \mathrm{O}_{2} \mathrm{mg}^{-1} \mathrm{WM} \mathrm{h}^{-1}$ in the intermediate size class of Primno macropa during winter to $0.225 \mu \mathrm{O}_{2} \mathrm{mg}^{-1} \mathrm{WM} \mathrm{h}^{-1}$ in the smallest size class of P. macropa during fall (Table 2). Rates for the hyperiid amphipods tended to be higher on average than those of the gammarids ( $\vec{x}=0.121$ vs $0.072 \mu \mathrm{O}_{2} \mathrm{mg}^{-1} \mathrm{WM} \mathrm{h}^{-1}$ ). The difference was at least partially due to the fact that hyperiids also tend to be smaller Rates normalized to $1 \mathrm{~g}$ showed less of a difference: $0.074 \mu \mathrm{O}_{2} \mathrm{mg}^{-1} \mathrm{WM} \mathrm{h}^{-1}$ for the hyperiids vs 0.054 for the gammarids (see Table 5). The ratio of maximum to routine rate in the hyperilds was $1.76 \pm$ $0.27(\bar{x} \pm \mathrm{SD})$; maximum to minimum ratio was $4.40 \pm$ 2.54. Pc-values were similar to those of the gammarids, ranging from a low of $29 \mathrm{~mm} \mathrm{Hg} \mathrm{O}_{2}$ in Vibilia stebbingi to $41 \mathrm{~mm} \mathrm{Hg} \mathrm{O}_{2}$ in Cyllopus lucasi. 
Table 2. Oxygen consumption rates of hyperiid amphipods. All values expressed as mean (SD). See Table 1 for abbreviations

\begin{tabular}{|c|c|c|c|c|c|c|c|c|c|c|}
\hline Species (n) & Season & $\begin{array}{l}\mathrm{MDO} \\
(\mathrm{m})\end{array}$ & $\begin{array}{c}\text { Mean } \\
\text { wet mass } \\
(\mathrm{g})\end{array}$ & $\begin{array}{l}\text { Size } \\
\text { class }\end{array}$ & $\begin{array}{l}\text { Oxygen } \\
\left(\mu l \mathrm{O}_{2}\right. \\
\text { Routine }\end{array}$ & $\begin{array}{c}\text { onsump } \\
\text { ng }^{-1} \text { WN } \\
\text { Min. }\end{array}$ & $\begin{array}{c}\text { tion rate } \\
\mathrm{M} \mathrm{h}^{-1} \text { ) } \\
\text { Max. }\end{array}$ & $\begin{array}{c}\mathrm{Pc} \\
(\mathrm{mm} \mathrm{Hg})\end{array}$ & $\begin{array}{l}\text { Conv } \\
\text { DM }\end{array}$ & $\begin{array}{l}\text { ersions } \\
\text { AFDM }\end{array}$ \\
\hline Cyllopus lucasii (4) & $\mathrm{F}$ & 0 & $\begin{array}{c}0.048 \\
(0.008)\end{array}$ & 3 & $\begin{array}{c}0.200 \\
(0.047)\end{array}$ & $\begin{array}{c}0.076 \\
(0.032)\end{array}$ & $\begin{array}{c}0.328 \\
(0.135)\end{array}$ & & 3.19 & 3.82 \\
\hline C. lucasii (1) & W & 0 & 0.043 & 3 & 0.175 & 0.015 & 0.317 & & 4.46 & 5.64 \\
\hline C. lucasii (2) & $\mathrm{S}$ & 0 & $\begin{array}{c}0.149 \\
(0.021)\end{array}$ & 5 & $\begin{array}{c}0.210 \\
(0.047)\end{array}$ & $\begin{array}{c}0.189 \\
(0.070)\end{array}$ & $\begin{array}{c}0.354 \\
(0.115)\end{array}$ & 41 & 2.75 & 3.24 \\
\hline C. lucasii (5) & $\mathrm{F}$ & 0 & $\begin{array}{c}0.197 \\
(0.059)\end{array}$ & 5 & $\begin{array}{c}0.149 \\
(0.023)\end{array}$ & $\begin{array}{c}0.119 \\
(0.015)\end{array}$ & $\begin{array}{c}0.353 \\
(0.059)\end{array}$ & 31 & 3.19 & 3.82 \\
\hline C. lucasii (6) & W & 0 & $\begin{array}{c}0.189 \\
(0.019)\end{array}$ & 5 & $\begin{array}{c}0.115 \\
(0.023)\end{array}$ & $\begin{array}{c}0.050 \\
(0.039)\end{array}$ & $\begin{array}{c}0.175 \\
(0.029)\end{array}$ & & 4.46 & 5.64 \\
\hline Hyperoche medusarum (2) & $\mathrm{F}$ & 0 & $\begin{array}{c}0.072 \\
(0.002)\end{array}$ & 4 & $\begin{array}{c}0.105 \\
(0.012)\end{array}$ & $\begin{array}{c}0.027 \\
(0.004)\end{array}$ & $\begin{array}{c}0.210 \\
(0.007)\end{array}$ & & 5.39 & 6.86 \\
\hline Primno macropa (3) & $\mathrm{F}$ & 50 & $\begin{array}{c}0.034 \\
(0.014)\end{array}$ & 3 & $\begin{array}{c}0.225 \\
(0.010)\end{array}$ & $\begin{array}{c}0.121 \\
(0.036)\end{array}$ & $\begin{array}{c}0.388 \\
(0.051)\end{array}$ & 35 & 3.40 & 4.32 \\
\hline P. macropa (1) & W & 50 & 0.026 & 3 & 0.214 & 0.019 & 0.558 & & 4.25 & 5.38 \\
\hline P. macropa (10) & $\mathrm{F}$ & 50 & $\begin{array}{c}0.061 \\
(0.008)\end{array}$ & 4 & $\begin{array}{c}0.154 \\
(0.060)\end{array}$ & $\begin{array}{c}0.061 \\
(0.034)\end{array}$ & $\begin{array}{c}0.268 \\
(0.078)\end{array}$ & 32 & 3.40 & 4.32 \\
\hline P. macropa (1) & W & 50 & 0.056 & 4 & 0.051 & 0.006 & 0.108 & & 4.25 & 5.38 \\
\hline P. macropa (6) & $\mathrm{F}$ & 50 & $\begin{array}{c}0.129 \\
(0.007)\end{array}$ & 5 & $\begin{array}{c}0.148 \\
(0.045)\end{array}$ & $\begin{array}{c}0.086 \\
(0.031)\end{array}$ & $\begin{array}{c}0.234 \\
(0.072)\end{array}$ & 37 & 3.40 & 4.32 \\
\hline Themisto gaudichaudi (2) & W & 0 & $\begin{array}{c}0.091 \\
(0.003)\end{array}$ & 4 & $\begin{array}{c}0.133 \\
(0.016)\end{array}$ & $\begin{array}{c}0.034 \\
(0.004)\end{array}$ & $\begin{array}{c}0.211 \\
(0.029)\end{array}$ & & 4.73 & 5.81 \\
\hline T. gaudichaudi (2) & $w$ & 0 & $\begin{array}{c}0.314 \\
(0.031)\end{array}$ & 5 & $\begin{array}{c}0.054 \\
(0.013)\end{array}$ & $\begin{array}{c}0.010 \\
(0.007)\end{array}$ & $\begin{array}{c}0.109 \\
(0.001)\end{array}$ & & 5.19 & 6.87 \\
\hline Vibilia stebbingi (4) & F & 0 & $\begin{array}{c}0.048 \\
(0.010)\end{array}$ & 3 & $\begin{array}{c}0.166 \\
(0.026)\end{array}$ & $\begin{array}{c}0.115 \\
(0.027)\end{array}$ & $\begin{array}{c}0.277 \\
(0.040)\end{array}$ & 29 & 4.18 & 5.29 \\
\hline V. stebbingi (8) & w & 0 & $\begin{array}{c}0.033 \\
(0.010)\end{array}$ & 3 & $\begin{array}{c}0.088 \\
(0.027)\end{array}$ & $\begin{array}{c}0.041 \\
(0.026)\end{array}$ & $\begin{array}{c}0.181 \\
(0.056)\end{array}$ & & 4.33 & 5.25 \\
\hline$V$. stebbingi (2) & $\mathrm{F}$ & 0 & $\begin{array}{c}0.117 \\
(0.003)\end{array}$ & 5 & $\begin{array}{c}0.148 \\
(0.004)\end{array}$ & $\begin{array}{c}0.078 \\
(0.010)\end{array}$ & $\begin{array}{c}0.202 \\
(0.002)\end{array}$ & 35 & 4.80 & 6.34 \\
\hline
\end{tabular}

Sufficient data were available in 1 size class to test for the effects of season in 2 species, Cyllopus lucasi and Vibilia stebbingi. C. lucasi showed a significant decline from spring to fall and from fall to winter $(p<0.05$, ANOVA). V. stebbingi showed a significant decline from fall to winter $(p<0.05$, ANOVA).

The relation of metabolism $\left(y, \mathrm{ml}\right.$ ind. $\left.{ }^{-1} \mathrm{~h}^{-1}\right)$ to mass $(x, g)$ in Primno macropa for the fall season was described by the equation: $y=0.401 x^{0.773 \pm 0.157}(b \pm S E$, $\left.\mathrm{r}^{2}=0.585, \mathrm{p}<0.001\right)$, suggesting that mass-specific metabolism declines with increasing mass.

The hyperiid species shown in Table 2 are widely distributed in the upper $500 \mathrm{~m}$ of the water column; their vertical profile virtually always includes a strong component in near-surface waters (Lancraft et al. 1989, 1991), which precludes any analysis for effects of depth of occurrence. Themisto gaudichaudii excepted, they are most similar to the sympagic gammarid amphipod Eusirus antarcticus in size and routine rate.

\section{Euphausiids}

Routine rates varied from $0.062 \mu \mathrm{l} \mathrm{O} \mathrm{mg}^{-1} \mathrm{WM} \mathrm{h}^{-1}$ in the largest size class of Euphausia superba during winter to $0.250 \mu \mathrm{l} \mathrm{O}_{2} \mathrm{mg}^{-1} \mathrm{WM} \mathrm{h}^{-1}$ in size class 3 during fall (Table 3). Rates for E. triacantha (0.101 to $0.127 \mathrm{\mu l} \mathrm{O}_{2} \mathrm{mg}^{-1} \mathrm{WM} \mathrm{h}^{-1}$ ) and Thysanoessa macrura $\left(0.123\right.$ to $\left.0.216 \mu \mathrm{O}_{2} \mathrm{mg}^{-1} \mathrm{WM} \mathrm{h}^{-1}\right)$ fell in the midrange of these values (Table 3 ). Ratios of maximum to routine rates $(1.81 \pm 0.37)$ and maximum to minimum rates $(5.56 \pm 3.40)$ were similar to those of the amphipods. Pc-values were obtained for a variety of size classes of E. superba and ranged from 30 to $52 \mathrm{~mm} \mathrm{Hg}$ $\mathrm{O}_{2}$. Pc values for $T$. macrura were similar, ranging from 32 to $40 \mathrm{~mm} \mathrm{Hg} \mathrm{O}$. Like the amphipods, aerobic respiration in euphausiids is not limited by ambient $\mathrm{PO}_{2}$.

Sufficient data were available to examine the effects of season on routine metabolism in Euphausia superba. Two methods of comparison were used. In the first, the 
Table 3. Oxygen consumption rates of euphausiids. All values expressed as mean (SD). See Table 1 for abbreviations

\begin{tabular}{|c|c|c|c|c|c|c|c|c|c|c|}
\hline \multirow{3}{*}{$\begin{array}{l}\text { Species (n) } \\
\text { Euphausia superba (9) }\end{array}$} & \multirow{3}{*}{$\begin{array}{l}\text { Season } \\
\qquad \mathrm{W}\end{array}$} & \multirow{3}{*}{$\begin{array}{c}\mathrm{MDO} \\
(\mathrm{m}) \\
0\end{array}$} & \multirow{3}{*}{$\begin{array}{c}\text { Mean } \\
\text { wet mass } \\
(g) \\
0.003 \\
(0.001)\end{array}$} & \multirow{3}{*}{$\begin{array}{c}\text { Size } \\
\text { class } \\
1\end{array}$} & \multirow{2}{*}{\multicolumn{3}{|c|}{$\begin{array}{l}\text { Oxygen consumption rate } \\
\left(\mu \mathrm{O} \mathrm{O}_{2} \mathrm{mg}^{-1} \mathrm{WM} \mathrm{h}^{-1}\right)\end{array}$}} & \multirow{2}{*}{$\begin{array}{c}\mathrm{Pc} \\
(\mathrm{mm} \mathrm{Hg})\end{array}$} & \multicolumn{2}{|c|}{ Conversions } \\
\hline & & & & & & & & & \multicolumn{2}{|c|}{ DM AFDM } \\
\hline & & & & & $\begin{array}{c}0.171 \\
(0.037)\end{array}$ & $\begin{array}{c}0.046 \\
(0.043)\end{array}$ & $\begin{array}{c}0.312 \\
(0.084)\end{array}$ & & 3.38 & 3.73 \\
\hline E. superba (6) & W & 0 & $\begin{array}{c}0.006 \\
(0.001)\end{array}$ & 2 & $\begin{array}{c}0.146 \\
(0.029)\end{array}$ & $\begin{array}{l}0.057 \\
(0.019)\end{array}$ & $\begin{array}{c}0.343 \\
(0.124)\end{array}$ & 52 & 3.44 & 3.86 \\
\hline E. superba (1) & $\mathrm{S}$ & 0 & 0.017 & 3 & 0.229 & 0.097 & 0.265 & & 3.68 & 4.36 \\
\hline E. superba (4) & $F$ & 0 & $\begin{array}{c}0.036 \\
(0.007)\end{array}$ & 3 & $\begin{array}{c}0.250 \\
(0.066)\end{array}$ & $\begin{array}{c}0.062 \\
(0.063)\end{array}$ & $\begin{array}{l}0.357 \\
(0.045)\end{array}$ & & 2.90 & 3.26 \\
\hline E. superba (2) & W & 0 & $\begin{array}{c}0.017 \\
(0.000)\end{array}$ & 3 & $\begin{array}{c}0.248 \\
(0.026)\end{array}$ & $\begin{array}{l}0.030 \\
(0.008)\end{array}$ & $\begin{array}{c}0.446 \\
(0.021)\end{array}$ & & 3.37 & 3.91 \\
\hline E. superba (2) & $\mathrm{F}$ & 0 & $\begin{array}{c}0.060 \\
(0.000)\end{array}$ & 4 & $\begin{array}{c}0.159 \\
(0.033)\end{array}$ & $\begin{array}{c}0.062 \\
(0.039)\end{array}$ & $\begin{array}{l}0.357 \\
(0.045)\end{array}$ & 42 & 3.25 & 3.69 \\
\hline E. superba (1) & W & 0 & 0.056 & 4 & 0.105 & 0.041 & 0.217 & & 3.33 & 3.68 \\
\hline E. superba (3) & $\mathrm{S}$ & 0 & $\begin{array}{c}0.413 \\
(0.107)\end{array}$ & 5 & $\begin{array}{c}0.133 \\
(0.014)\end{array}$ & $\begin{array}{c}0.065 \\
(0.023)\end{array}$ & $\begin{array}{c}0.211 \\
(0.036)\end{array}$ & 42 & 3.68 & 4.36 \\
\hline E. superba (3) & $\mathrm{F}$ & 0 & $\begin{array}{c}0.382 \\
(0.039)\end{array}$ & 5 & $\begin{array}{c}0.152 \\
(0.057)\end{array}$ & $\begin{array}{c}0.067 \\
(0.063)\end{array}$ & $\begin{array}{c}0.242 \\
(0.050)\end{array}$ & & 4.53 & 5.23 \\
\hline E. superba (27) & W & 0 & $\begin{array}{c}0.280 \\
(0.112)\end{array}$ & 5 & $\begin{array}{c}0.071 \\
(0.019)\end{array}$ & $\begin{array}{c}0.018 \\
(0.011)\end{array}$ & $\begin{array}{c}0.148 \\
(0.057)\end{array}$ & 30 & 4.07 & 4.67 \\
\hline E. superba (7) & $\mathrm{S}$ & 0 & $\begin{array}{c}0.780 \\
(0.155)\end{array}$ & 6 & $\begin{array}{c}0.141 \\
(0.027)\end{array}$ & $\begin{array}{c}0.097 \\
(0.026)\end{array}$ & $\begin{array}{c}0.199 \\
(0.069)\end{array}$ & 44 & 3.68 & 4.36 \\
\hline E. superba (5) & $F$ & 0 & $\begin{array}{c}0.701 \\
(0.104)\end{array}$ & 6 & $\begin{array}{c}0.166 \\
(0.064)\end{array}$ & $\begin{array}{c}0.099 \\
(0.049)\end{array}$ & $\begin{array}{c}0.248 \\
(0.090)\end{array}$ & 35 & 3.43 & 3.72 \\
\hline E. superba (17) & W & 0 & $\begin{array}{c}0.618 \\
(0.071)\end{array}$ & 6 & $\begin{array}{c}0.067 \\
(0.021)\end{array}$ & $\begin{array}{c}0.021 \\
(0.012)\end{array}$ & $\begin{array}{c}0.153 \\
(0.050)\end{array}$ & & 4.63 & 5.38 \\
\hline E. superba (2) & $\mathrm{S}$ & 0 & $\begin{array}{c}1.118 \\
(0.068)\end{array}$ & 7 & $\begin{array}{c}0.174 \\
(0.004)\end{array}$ & $\begin{array}{c}0.122 \\
(0.013)\end{array}$ & $\begin{array}{c}0.261 \\
(0.017)\end{array}$ & & 3.68 & 4.36 \\
\hline E. superba (1) & $w$ & 0 & 1.120 & 7 & 0.062 & 0.033 & 0.118 & & 3.68 & 4.09 \\
\hline E. triacantha (5) & $w$ & 50 & $\begin{array}{c}0.058 \\
(0.019)\end{array}$ & 4 & $\begin{array}{c}0.127 \\
(0.015)\end{array}$ & $\begin{array}{c}0.055 \\
(0.030)\end{array}$ & $\begin{array}{c}0.226 \\
(0.049)\end{array}$ & & 3.94 & 4.66 \\
\hline E. triacantha (2) & W & 50 & $\begin{array}{c}0.154 \\
(0.007)\end{array}$ & 5 & $\begin{array}{c}0.101 \\
(0.025)\end{array}$ & $\begin{array}{c}0.062 \\
(0.021)\end{array}$ & $\begin{array}{c}0.138 \\
(0.042)\end{array}$ & & 3.90 & 4.70 \\
\hline Thysanoessa macrura (10) & W & 50 & $\begin{array}{c}0.033 \\
(0.006)\end{array}$ & 3 & $\begin{array}{c}0.179 \\
(0.035)\end{array}$ & $\begin{array}{c}0.038 \\
(0.023)\end{array}$ & $\begin{array}{c}0.400 \\
(0.072)\end{array}$ & & 3.25 & 3.61 \\
\hline T. macrura (1) & $\mathrm{F}$ & 100 & 0.075 & 4 & 0.216 & 0.030 & 0.381 & & 2.86 & 3.12 \\
\hline T. macrura $(10)$ & W & 50 & $\begin{array}{c}0.071 \\
(0.011)\end{array}$ & 4 & $\begin{array}{c}0.145 \\
(0.045)\end{array}$ & $\begin{array}{c}0.058 \\
(0.030)\end{array}$ & $\begin{array}{l}0.276 \\
(0.084)\end{array}$ & 32 & 3.37 & 3.81 \\
\hline T.macrura $(6)$ & W & 50 & $\begin{array}{c}0.129 \\
(0.029)\end{array}$ & 5 & $\begin{array}{c}0.120 \\
(0.015)\end{array}$ & $\begin{array}{c}0.051 \\
(0.023)\end{array}$ & $\begin{array}{l}0.259 \\
(0.099)\end{array}$ & 40 & 3.53 & 4.12 \\
\hline
\end{tabular}

size class for which the greatest quantity of data were available (size class 6) was compared across all 3 seasons. Spring and fall data were not significantly different $(p>0.05, \text { ANOVA })_{i}$ these were lumped and compared to winter data using ANOVA. The mean routine rate for spring/fall $\left(0.152 \pm 0.022 \mu \mathrm{l} \mathrm{O}_{2} \mathrm{mg}^{-1}\right.$ $\mathrm{WM} \mathrm{h}^{-1} ; \bar{x} \pm 95 \% \mathrm{CL}$ ) was significantly different from the winter rate $\left(0.066 \pm 0.018 \mathrm{ul} \mathrm{O}_{2} \mathrm{mg}^{-1} \mathrm{WM} \mathrm{h}^{-1}\right)$ $(p<0.001$, ANOVA). The second comparison that was made was of the regression lines of routine rate versus mass for the spring/fall data and the winter data using ANCOVA (Fig. 1). ANCOVA compares 3 properties of regression lines: the residual variances, the slope, and the elevation of the lines. The residual variances and slopes were not significantly different $(p>0.05)$ but the point of comparison most relevant to a lowered winter metabolic rate, the elevations of the lines, was significantly different $(p<0.05)$. Analysis of the seasonal data using both ANOVA and ANCOVA strongly suggests that metabolic rate is lowered in E. superba during the winter season. The difference is not subtle; winter metabolic rates are about $45 \%$ of those in the spring and fall in the larger size classes.

The relation of routine rate $\left(y, \mathrm{ml} \mathrm{O}_{2}\right.$ ind. $\left.^{-1} \mathrm{~h}^{-1}\right)$ to wet mass $(x, g)$ in Thysanoessa macrura during the winter season was described by the equation: $y=$ 
$0.352 x^{0.786 \pm 0098}\left(b \pm S E, r^{2}=0.727, p<0.001\right)$. The routine rate of $T$. macrura thus scales with mass in a conventional manner.

The euphausiids, like the hyperiids, are distributed throughout the upper $500 \mathrm{~m}$ of the water column with a large fraction of the population found in surface waters, particularly in Euphausia superba. Their routine rates are similar to the shallow-dwelling hyperiids and gammarids.

\section{Decapods}

Routine rates in the decapods showed little variability between species. Petalidium foliaceum had the lowest rate $\left(0.036 \mu \mathrm{O}_{2} \mathrm{mg}^{-1}\right.$ $\mathrm{WM} \mathrm{h} \mathrm{h}^{-1}$ ) and Gennadas kempi the highest $\left(0.056 \mu \mathrm{l} \mathrm{O}_{2} \mathrm{mg}^{-1} \mathrm{WM} \mathrm{h}^{-1}\right)$ (Table 4). Ratios of maximum to routine rate $(2.64 \pm 0.58)$ were similar to those observed in the other groups; maximum to minimum were somewhat higher $(9.24 \pm 4.67)$.

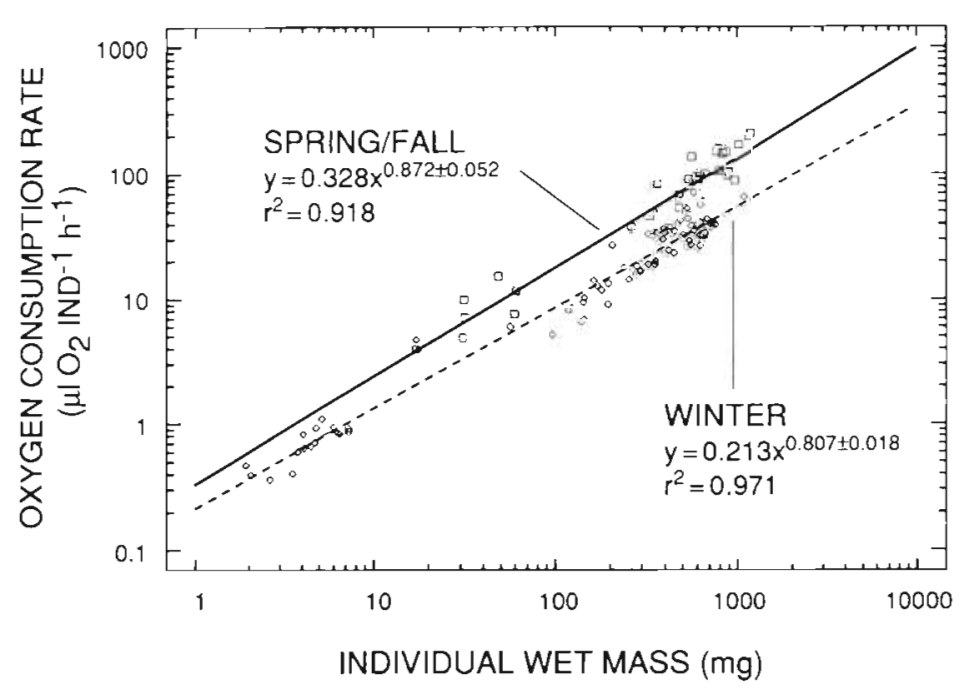

Fig. 1. Oxygen consumption rate of Euphausia superba as a function of season. (ㅁ) Spring/fall values; ( $(0)$ winter values. Slopes are expressed \pm standard error. Regressions were fitted using the least squares method; lines are significantly different $(p<0.01$, ANCOVA)

Table 4. Oxygen consumption rates of decapods, isopods, mysids and ostracods. All values expressed as mean (SD). See Table 1 for abbreviations

\begin{tabular}{|c|c|c|c|c|c|c|c|c|c|c|}
\hline $\begin{array}{l}\text { Group } \\
\quad \text { Species (n) }\end{array}$ & Season & $\begin{array}{l}\mathrm{MDO} \\
(\mathrm{m})\end{array}$ & $\begin{array}{c}\text { Mean } \\
\text { wet mass } \\
(\mathrm{g})\end{array}$ & $\begin{array}{l}\text { Size } \\
\text { class }\end{array}$ & $\begin{array}{r}\text { Oxygen } \\
\left(\mu l \mathrm{O}_{2}\right. \\
\text { Routine }\end{array}$ & $\begin{array}{c}\text { onsumpt } \\
\mathrm{ng}^{-1} \text { WM } \\
\text { Min. }\end{array}$ & $\begin{array}{l}\text { tion rate } \\
\mathrm{M} \mathrm{h}^{-1} \text { ) } \\
\text { Max. }\end{array}$ & $\begin{array}{c}\mathrm{Pc} \\
(\mathrm{mm} \mathrm{Hg})\end{array}$ & $\begin{array}{l}\text { Conv } \\
\text { DM }\end{array}$ & $\begin{array}{l}\text { ersions } \\
\text { AFDM }\end{array}$ \\
\hline \multicolumn{11}{|l|}{ Decapoda } \\
\hline Gennadas kempi (1) & W & 1000 & 0.231 & 5 & 0.051 & 0.014 & 0.101 & & 3.91 & 4.19 \\
\hline G. kempi (3) & w & 1000 & $\begin{array}{l}1.039 \\
(0.111)\end{array}$ & 7 & $\begin{array}{c}0.056 \\
(0.010)\end{array}$ & $\begin{array}{c}0.008 \\
(0.005)\end{array}$ & $\begin{array}{c}0.101 \\
(0.026)\end{array}$ & & 3.53 & 3.85 \\
\hline Petalidium foliaceum (2) & W & 1000 & $\begin{array}{c}1.164 \\
(0.206)\end{array}$ & 7 & $\begin{array}{c}0.036 \\
(0.019)\end{array}$ & $\begin{array}{c}0.009 \\
(0.006)\end{array}$ & $\begin{array}{c}0.100 \\
(0.058)\end{array}$ & & 3.30 & 3.57 \\
\hline Pasiphaea scotiae (2) & $\mathrm{S}$ & 100 & $\begin{array}{c}5.471 \\
(1.563)\end{array}$ & 8 & $\begin{array}{c}0.037 \\
(0.004)\end{array}$ & $\begin{array}{c}0.021 \\
(0.004)\end{array}$ & $\begin{array}{c}0.049 \\
(0.006)\end{array}$ & & 3.02 & 3.16 \\
\hline P. scotiae (3) & w & 1000 & $\begin{array}{c}1.898 \\
(1.058)\end{array}$ & 7 & $\begin{array}{c}0.043 \\
(0.005)\end{array}$ & $\begin{array}{c}0.010 \\
(0.003)\end{array}$ & $\begin{array}{c}0.109 \\
(0.042)\end{array}$ & & 2.59 & 2.82 \\
\hline \multicolumn{11}{|l|}{ Isopoda } \\
\hline Anuropus australis (1) & $\mathrm{S}$ & 100 & 0.772 & 6 & 0.025 & 0.007 & 0.043 & & 6.60 & 8.30 \\
\hline A. australis (1) & $F$ & 100 & 0.714 & 6 & 0.042 & 0.006 & 0.087 & & 6.60 & 8.30 \\
\hline A. australis (1) & $\mathrm{S}$ & nd & 2.258 & 7 & 0.029 & 0.008 & 0.041 & & 6.60 & 8.30 \\
\hline \multicolumn{11}{|l|}{ Mysidacea } \\
\hline Gnathophausia gigas (2) & $S$ & 1000 & $\begin{array}{c}0.503 \\
(0.250)\end{array}$ & 6 & $\begin{array}{c}0.053 \\
(0.011)\end{array}$ & $\begin{array}{c}0.009 \\
(0.001)\end{array}$ & $\begin{array}{c}0.059 \\
(0.007)\end{array}$ & & 2.72 & 4.17 \\
\hline G. gigas (1) & W & 1000 & 0.363 & 5 & 0.041 & 0.027 & 0.050 & & 4.00 & 4.64 \\
\hline G. gigas (2) & W & 1000 & $\begin{array}{c}6.321 \\
(0.138)\end{array}$ & 8 & $\begin{array}{c}0.038 \\
(0.003)\end{array}$ & $\begin{array}{c}0.013 \\
(0.007)\end{array}$ & $\begin{array}{c}0.069 \\
(0.021)\end{array}$ & & 3.65 & 4.61 \\
\hline \multicolumn{11}{|l|}{ Ostracoda } \\
\hline Gigantocypris mulleri (2) & ) $S$ & 500 & $\begin{array}{c}0.594 \\
(0.002)\end{array}$ & 6 & $\begin{array}{c}0.016 \\
(0.001)\end{array}$ & $\begin{array}{c}0.007 \\
(0.005)\end{array}$ & $\begin{array}{c}0.029 \\
(0.004)\end{array}$ & & 10.00 & 12.50 \\
\hline G. mulleri (2) & $\mathrm{S}$ & 500 & $\begin{array}{c}1.220 \\
(0.044)\end{array}$ & 7 & $\begin{array}{c}0.012 \\
(0.003)\end{array}$ & $\begin{array}{c}0.007 \\
(0.003)\end{array}$ & $\begin{array}{c}0.020 \\
(0.003)\end{array}$ & 30 & 10.00 & 12.60 \\
\hline G. mulleri (2) & W & 500 & $\begin{array}{c}1.690 \\
(0.558)\end{array}$ & 7 & $\begin{array}{c}0.009 \\
(0.002)\end{array}$ & $\begin{array}{c}0.002 \\
(0.001)\end{array}$ & $\begin{array}{c}0.026 \\
(0.004)\end{array}$ & & 13.18 & 17.43 \\
\hline
\end{tabular}




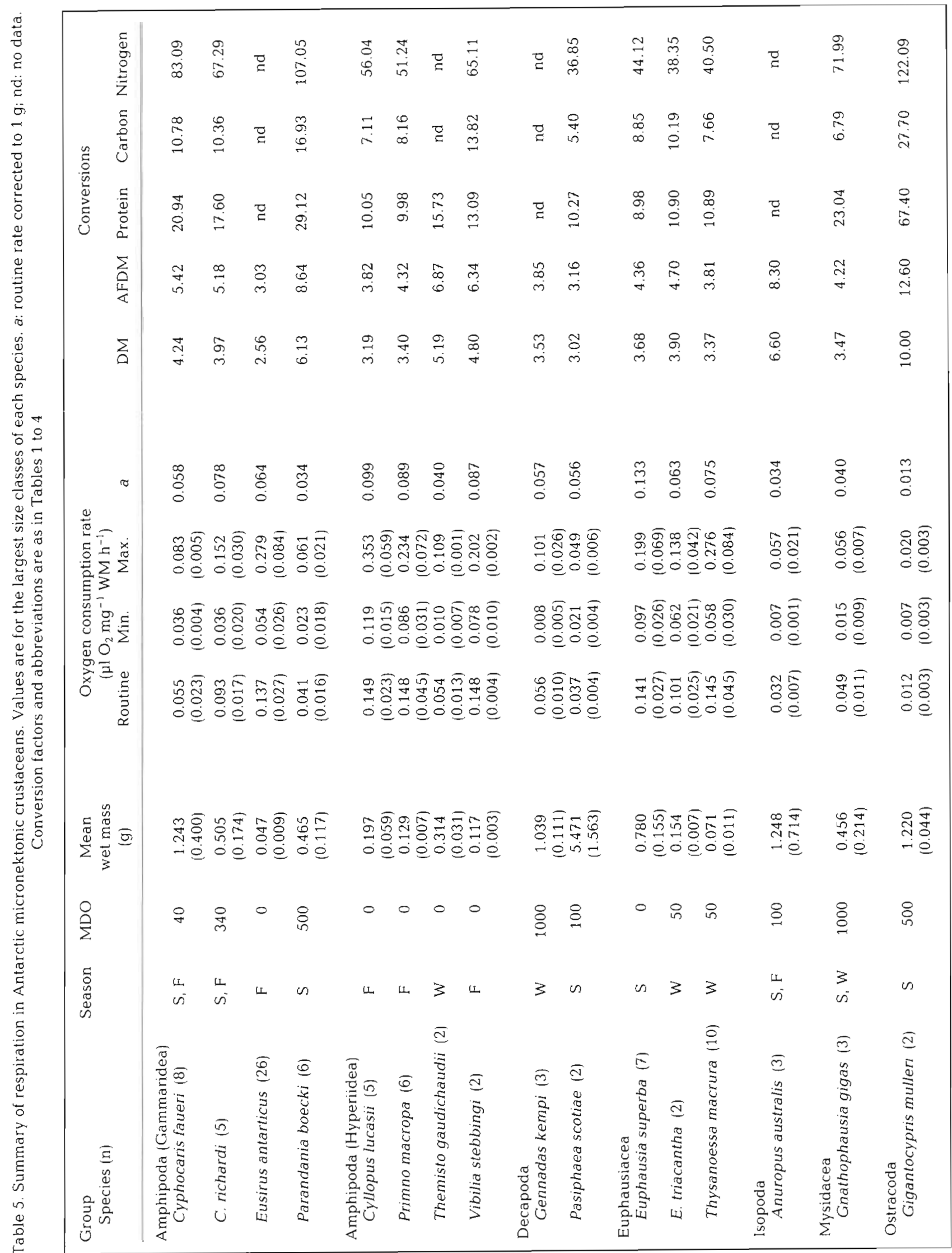


The decapods as a group are found at greater depths than the majority of the amphipods and euphausiids (Table 5) and they also are larger; both conditions result in a lower mass-specific metabolism. If rates normalized to $1 \mathrm{~g}$ are compared, the average value for the decapods $(0.050)$ is similar to that of the gammarids $(0.054)$, but lower than that of the euphausiids $(0.090)$ and hyperiids $(0.074)$.

\section{Isopods, mysids, and ostracods}

Each of these 3 orders is represented by 1 species. The giant ostracod, Gigantocypris mulleri, shows the lowest routine rate of the 3 and of the entire suite of Antarctic mesopelagic Crustacea shown in Tables 1 to 4: $0.009 \mu l \mathrm{O}_{2} \mathrm{mg}^{-1} \mathrm{WM} \mathrm{h}^{-1}$ (Table 4). The highest rate of the 3 is exhibited by Gnathophausia gigas: $0.053 \mu \mathrm{O} \mathrm{O}_{2} \mathrm{mg}^{-1} \mathrm{WM} \mathrm{h}^{-1}$. The rates of the 3 species are most similar to the decapods, which have a similar depth distribution, and to the deeper dwelling gammarids (Table 5).

\section{Overall trends}

The relation of routine rate $\left(y, \mu l \mathrm{O}_{2} \mathrm{mg}^{-1} \mathrm{WM} \mathrm{h}^{-1}\right)$ to depth of occurrence $(x, m)$ for the entire data set is described by the equation: $y=0.125 x^{-0172 \pm 0052}$ $(b \pm \mathrm{SE}), \mathrm{r}^{2}=0.434, \mathrm{p}<0.05$. There is also a significant decline of metabolism with depth in rates expressed as a function of dry mass $(y=$ $\left.0.461 x^{-0135 \pm 0.037}, r^{2}=0.477, p<0.05\right)$ and ash-free dry mass $\left(y=0.563 x^{-0.137 \pm 0.039}, r^{2}=0.471, p<0.05\right)$, although the slope in the latter 2 cases is only $78 \%$ that of the routine rate. If routine rates are normalized to $1 \mathrm{~g}$ to ameliorate the effects of increasing mass with depth ( $a$ in Table 5), the resulting line is described by the equation: $y=0.0820 x^{-0.113+0.042}, r^{2}=0.331, p<$ 0.05 . Similarly, if normalized rates are expressed as dry mass $\left(y=0.300 x^{-0.073 \pm 0.029}, \mathrm{r}^{2}=0.318, \mathrm{p}<0.05\right)$ or ash-free dry mass $\left(y=0.372 x^{-0.076 \pm 0.030}, \mathrm{r}^{2}=0.307, \mathrm{p}<\right.$ 0.05 ) the decline is still significant with a slope $67 \%$ of that for the routine rates. Thus, about $35 \%$ of the observed decline in rates may be ascribed to increased mass in deeper-living species; 20 to $30 \%$ may be assigned to the increased water content of deeper species.

The $b$-value used to normalize masses to $1 \mathrm{~g}, 0.75$, was chosen because it is the most widely accepted slope $(b)$ in the equation describing the relationship of metabolism $(y)$ and mass $(M): y=a M^{b}$, where $a$ is a proportionality constant (cf. Burggren \& Roberts 1991). However, the choice of $b$ clearly influences the contribution of mass to the decline in metabolism with depth. A $b$-value of 1.0 would erase any contribution of mass to the depth-related decline; a $b$-value of 0.67 would accentuate it. For purposes of comparing metabolism over the different taxonomic categories presented here it was deemed most important to treat all species the same way, hence the use of the historically accepted figure of 0.75 for normalizing metabolism to a mass of $1 \mathrm{~g}$. With our currently imperfect understanding of the influence of mass on metabolism (cf. Heusner 1982, 1987), this approach was a more reasonable course of action than ignoring the influence of mass altogether, since it does increase with increasing depth.

Data on chemical composition are available for 13 of the species (Table 5; Torres et al. 1994) which allows their rates to be expressed in terms of body carbon and body nitrogen (cf. Ikeda 1988). If mass-normalized data for the 13 species are analysed in a series of regressions similar to those described above, on a carbon-specific basis: $y=0.943 x^{-0.138 \pm 0.035}, r^{2}=0.578, p<$ 0.05 , or nitrogen-specific basis: $y=5.346 x^{-0.123 \pm 0.039}$, $r^{2}=0.482, p<0.05$, the decline of metabolism with depth remains significant.

Protein level ( $y, \mathrm{mg}$ protein $\mathrm{g}^{-1}$ AFDW) declines with depth $(x, m)$ in the suite of micronektonic crustacean species examined here according to the relationship: $y=x^{-0.082 \pm 0.024}, \mathrm{r}^{2}=0.506, \mathrm{p}<0.05$ (Torres et al. 1994). Protein-specific metabolism is approximately constant. The reduced metabolism in deeperliving species is thus partially explained through a reduction in the total muscle mass of deeper-living species rather than a change in the character of the muscle itself. This result contrasts with a similar analysis in fishes (Torres et al. 1979) in which deeperliving species showed a reduction in protein-specific metabolism with depth.

\section{DISCUSSION}

\section{Respiration and depth}

The relation of depth and metabolic rate observed in the present study is of particular interest due to the nearly isothermal character of the water column. Temperature varies by approximately $2^{\circ} \mathrm{C}$ in the upper $1000 \mathrm{~m}$ on an annual basis $\left(-1.87\right.$ to $0.25^{\circ} \mathrm{C}$; Gordon et al. 1982). Results from this study suggest that wet-mass-specific oxygen consumption rate declines significantly with depth such that the metabolism of a species living at $1000 \mathrm{~m}$ is about $30 \%$ that of a species living at the surface. The decline of metabolism with depth remains statistically intact whether the respiratory data are expressed as a function of dry mass, ash-free dry mass, carbon, or nitro- 
gen. If all data are mass-normalized to $1 \mathrm{~g}$ and expressed in terms of ash-free dry mass (or carbon or nitrogen), a species living at $1000 \mathrm{~m}$ respires at a rate about $60 \%$ that of a surface-dwelling species. The reduced metabolism may be considered an adapted characteristic of deeper-living species (cf. Childress 1971, 1975, Torres et al. 1979, Torres \& Somero 1988, Cowles et al. 1991).

In a similar study on Antarctic micronektonic Crustacea, Ikeda (1988) arrived at a similar conclusion regarding the magnitude of reduction in metabolism with depth in Antarctic species: deeper-living species show a metabolism about half that of species living in surface waters if size and water content are corrected for. However, the reduction in metabolism was attributed to a reduced activity level in deeper-living species. This is not the case. The ratio of maximum to minimum rates, which may be considered a primitive metabolic scope, shows no significant difference between species living at 0 or $1000 \mathrm{~m}$. A similar trend is observed in the ratio of maximum to routine rates. Deeper-living species are capable of modulating their activity level and their metabolic rates equivalently to those of surface dwellers. The respiratory rate itself is lower. Deep-living species are not surface-dwelling species that are less active; they are fundamentally different in character, exhibiting higher water levels, lower protein and lipid levels (Childress \& Nygaard 1973, 1974), lower metabolic rates (Childress 1975 , Torres et al. 1979), lowered activities of intermediary metabolic enzymes (Childress \& Somero 1979, Torres \& Somero 1988) insensitivity to pressure (Somero 1992) and a variety of other specializations (reviewed in Somero 1991)

\section{Regulation of oxygen consumption: PC}

Data on the critical oxygen partial pressure were obtained for 9 of the 21 species shown in Tables 1 to 4 . Values fell between 29 and $52 \mathrm{~mm} \mathrm{Hg} \mathrm{O}$, well below the lowest $\mathrm{PO}_{2}$ values found in the water column of the Scotia-Weddell Sea region (ca $100 \mathrm{~mm} \mathrm{Hg}$; Gordon et al. 1982). The Pc-values obtained for Antarctic Crustacea are in the same range as those obtained for pelagic Crustacea of the Gulf of Mexico $(20$ to $40 \mathrm{~mm}$ Hg; Donnelly \& Torres 1988) and those off Hawaii (23 to $35 \mathrm{~mm} \mathrm{Hg}$; Cowles et al. 1991) but much higher than those from the California borderland $(4.5$ to $20.5 \mathrm{~mm}$ $\mathrm{Hg}$; Childress 1975), which has a severe oxygen minimum layer. It thus appears that the low Pc-values observed in oxygen minimum layer species are a specific adaptation to that type of oxygen profile. Low PCvalues are not a general characteristic of mesopelagic crustacean species.

\section{Effects of season}

Three species showed a significant drop in metabolism in the winter months: Euphausia superba, $C y l$ lopus lucasii, and Vibilia stebbingi. In each of the species the drop was accompanied by a change in its chemical composition that would suggest starvation (Torres et al. 1994). In the case of E. superba and $C$. lucasii large increases in water level were noted in individuals collected in winter, accompanied by significant $(\mathrm{p}<0.05$, ANOVA) declines in protein level (E. superba) and in both protein and lipid (C. lucasil). $V$. stebbingi showed a large decline in protein level from fall to winter but the sample size in fall was too small $(n=1)$ for statistical comparison with winter data.

Each of the 3 species exhibiting a lower winter metabolic rate depend directly (Euphausia superba) or indirectly (the amphipods) on primary production for part of their diet. The hyperiid amphipods Cyllopus lucasii and Vibilia stebbingi are ectocommensals on salps (cf. Madin \& Harbison 1977). In our study area during the winter months, salps were very rare (Lancraft et al. 1991); consequently, both amphipod species were deprived of their preferred host. Vibilia spp. feeds primarily on particulate food collected by salps (Madin \& Harbison 1977) and is thus indirectly dependent on the primary production harvested by its host salp for its nutrition. In addition, the host salp provides a free ride for the amphipods, allowing them to eliminate much of the energy costs entailed in maintaining vertical position in the water column. The seasonal drop in salp populations thus hits the hyperiids with a 2-fold energy cost: less food plus the need to provide the energy for their own locomotion. The increased energy costs coupled with lowered food availability results in a depletion of body reserves and the lowered metabolic rate that often accompanies starvation (cf. Ikeda \& Dixon 1982)

The remaining species either show no significant change in oxygen consumption with season (Cyphocaris richardi, Parandania boecki, Pasiphaea scotiae) or data are insufficient to test for seasonal effects. Those species that are primarily carnivorous, which would include all the deeper-living species, would presumably be less severely impacted by the restricted primary production typical of the winter months

The lowered winter metabolic rate in Euphausia superba is unequivocal and is present throughout the wide range of masss tested in this study (Fig. 1). Even in the smallest sizes of E. superba a lowered metabolic rate is evident. Ikeda (1981) reports respiratory values of $0.969 \pm 0.208 \mu \mathrm{O}_{2} \mathrm{mg}^{-1} \mathrm{DW} \mathrm{h}{ }^{-1}$ for furcilia IV-V taken during the austral summer-fall in the ScotiaWeddell Sea region. Our winter values for furcilia IV $-\mathrm{V}$ (size classes 1 and 2) are approximately half 
$(0.541 \pm 0.112, \bar{x} \pm \mathrm{SD})$ those reported by Ikeda (1981). The phenomenon of a lowered winter metabolic rate in E. superba was first reported by Kawaguchi et al. (1986) in E. superba captured under coastal fast ice in Lutzow-Holm Bay. It has subsequently been reported for krill captured in the vicinity of Palmer Peninsula (Quetin \& Ross 1991) and now for a wide size range of krill captured in the Scotia-Weddell Sea region.

The lower rate of Euphasia superba can be purely a function of starvation, which is certainly in evidence for the suite of krill examined in this study, or it can be a form of dormancy elicited by the physical conditions of the austral winter. Ikeda \& Dixon (1984) report an increase in metabolism in krill with an increase in ingestion rate, but the magnitude of the effect is insufficient to explain the rate reduction observed in the winter krill. However, Ikeda \& Dixon (1982) report rates for starved krill that are identical with our winter rates. Preliminary data on RNA-DNA ratio (summer: $6.19 \pm 1.18$; winter: $1.45 \pm 0.382 ; \bar{x} \pm \mathrm{SD}$; Torres \& Somero unpubl. data) and activity of the metabolic enzyme citrate synthase (summer: $1.56 \pm 0.37 \mu \mathrm{mol}$ substrate converted to product $\mathrm{g}^{-1} \mathrm{WM} \mathrm{min}^{-1}$; winter: $0.274 \pm 0.221 ; \bar{x} \pm \mathrm{SD}$ ) show profound reductions in protein synthetic capacity and general metabolic activity in winter krill. Starvation is sufficient to produce the magnitude of rate reduction observed in winter krill, but other physiological mechanisms may be responsible as well.

\section{Comparison with other systems}

Six studies have now addressed the metabolism of micronektonic Crustacea in widely disparate regions of the world ocean. Two of the studies took place in the sub-Arctic transitional waters of the California borderland (Childress 1975, Quetin et al. 1980), one in the subtropical waters of the Gulf of Mexico (Donnelly \& Torres 1988), 1 in the subtropical waters of the Pacific central gyre near Hawaii (Cowles et al. 1991), and 2 in the polar waters of the Antarctic (Ikeda 1988, this paper). The taxonomic composition and life habits of the micronektonic Crustacea examined differ considerably between studies, mirroring the composition of the local fauna. However, a few generalizations can be made. Representation by the decapods increases with increasing sea-surface temperature at the study sites. Decapods comprised $13 \%$ of the species examined in the Antarctic (this paper) and $93 \%$ of those in the Gulf of Mexico. In the Pacific, decapods comprise 43 to $58 \%$ of the species examined in the California borderland and $71 \%$ of those examined off Hawaii. Representation by the amphipods decreases with increasing seasurface temperature with $50 \%$ of the data set from the
Antarctic comprised of hyperiids and gammarids; they are not represented at all in the Gulf of Mexico and vary from 5 to $10 \%$ in data from the Pacific. Mysids, ostracods, and isopods comprise from $0 \%$ of the data set in the Gulf of Mexico to $30 \%$ in Hawaiian waters, with the remainder falling between 16 and $25 \%$. The last major group, the euphausiids, vary from 4 to $7 \%$ in all data sets outside the Antarctic, where they comprise $19 \%$ of the species. The fraction of those species that perform a diel vertical migration represented in each data set scales roughly with sea-surface temperature, with $13 \%$ of the Antarctic species showing a diel migration and $100 \%$ of those in the Gulf of Mexico migrating towards the surface on a diel basis. The remainder fall between 21 (California borderland) and $41 \%$ (Hawaii). Deep-living ( $\geq 500 \mathrm{~m}$ MDO) nonmigrating species are most numerous in the 3 Pacific studies (58 to $65 \%$ of the species), least numerous in the Gulf of Mexico $(0 \%)$, and intermediate in the Antarctic (25\%). Data from all 6 studies, with rates normalized to a mass of $1 \mathrm{~g}$ using a $b$-value of 0.75 and to a temperature of $0.5^{\circ} \mathrm{C}$ using a $Q_{10}$ of 2.0 , are presented in Fig. 2

All of the 6 data sets show a trend of declining metabolism with increasing species' depth of occurrence in the mass- and temperature-corrected data (Fig. 2). In 2 data sets, the curve for the mass- and temperature-corrected data is not significant $(\mathrm{p}>0.05$, ANOVA: Quetin et al. 1980, Donnelly \& Torres 1988). In Donnelly \& Torres (1988), the data set is comprised entirely of species that migrate strongly over intermediate depths in the water column. The species all exhibit a conventional response to temperature $\left(Q_{10}=2.2\right.$ for crustaceans examined collectively), inhabit a considerable depth range (600 to $1000 \mathrm{~m}$ ), and possess shallow to intermediate MDOs (70 to $410 \mathrm{~m} ; 67 \%$ of species $\leq 200 \mathrm{~m}$ ). The species composition of the Gulf micronektonic Crustacea, the predominance of vertical migration as a foraging strategy, and the species' response to temperature result in a metabolic decline with depth largely due to temperature. When metabolic rates are normalized to $0.5^{\circ} \mathrm{C}$, the decline is no longer statistically significant. Within the Gulf of Mexico, deep-living non-migrating species found at depths of 600 to $1000 \mathrm{~m}$ in the Pacific or Antarctic (e.g. Acanthephyra acutifrons, Gnathophausia gracilis, G. gigas, Gigantocypris agassizii) are found predominantly below $1000 \mathrm{~m}$ (Hopkins et al. 1989, Hopkins unpubl. data). Much of the observed decline in metabolism with depth in the data sets from the Pacific and Antarctic is due to the low metabolism exhibited in the deep-living, non-migrating species groups. In the Gulf, the lower half of the upper $1000 \mathrm{~m}$ is occupied largely by deeper-living, strongly migrating species. 


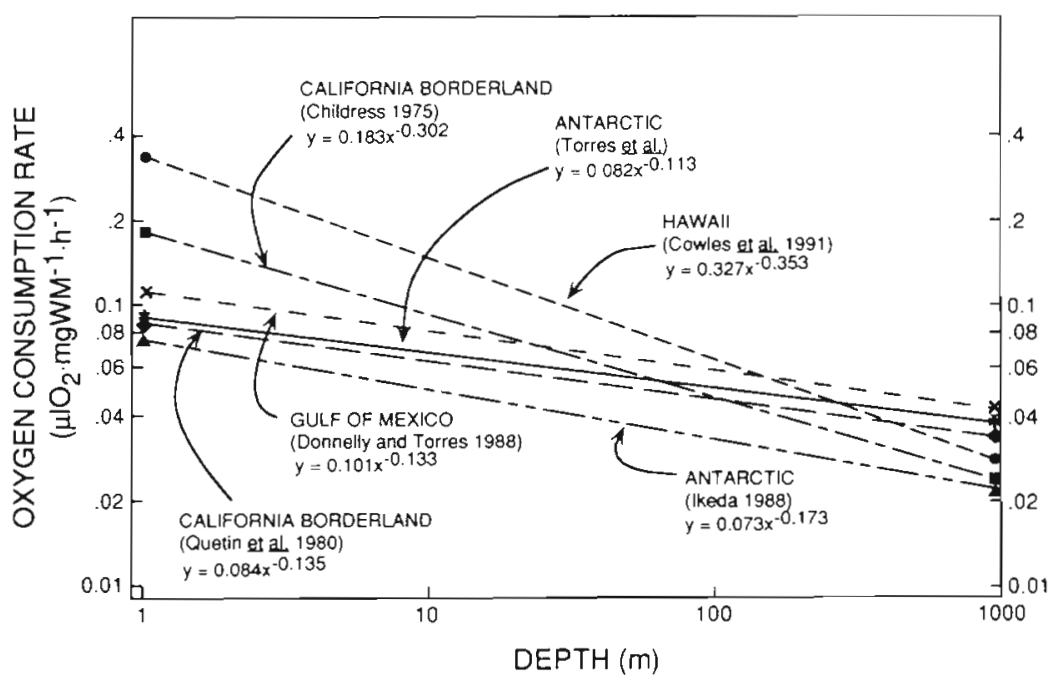

Fig. 2: Respiration versus depth in crustaceans from the Antarctic, California borderland, Gulf of Mexico, and central Pacific near Hawaii. All values corrected to a weight of $1 \mathrm{~g}$ using a $b$-value of 0.75 and to a temperature of $0.5^{\circ} \mathrm{C}$ using a $Q_{10}$ of 2.0

Variability in data sets from the same ocean system is best illustrated with the pair of studies from the California borderland (Childress 1975, Quetin et al. 1980) and from the Antarctic (Ikeda 1988, this paper). Different suites of species and different techniques were used by the investigators in each pair of studies. The data set of Quetin et al. (1980) when mass- and temperature-corrected does not show a significant decline with depth at the 0.05 level $(p=0.13)$ and differs from Childress (1975) in having a lower intercept and lesser slope (Fig. 2). The data set of Quetin et al. (1980) is a subset of that of Childress (1975) with less representation by deep-living non-migrating species. The differences between the 2 studies illustrate the range of variability to be expected within a system resulting from the suite of species examined and the techniques employed. Neither the slopes nor the intercepts are significantly different in the curves of Ikeda (1988) and the present study ( $p>0.05$ Student's $t)$, although Ikeda's curve lies below ours in Fig. 2 .

Two conclusions may be drawn by comparing the 6 curves in Fig. 2. The first is that the data do not support a case for metabolic temperature compensation among the micronektonic Crustacea in the sense of Scholander et al. (1953). The mass- and temperature-corrected curves generated for Antarctic species lie either well below or directly on top of those for lower latitude species. Absence of cold adaptation was also observed by Cowles et al. (1991) in comparing species from Hawaii and California waters. The second conclusion that may be drawn is that most of the variability between systems occurs in the top $200 \mathrm{~m}$ of the water column. Convergence of the curves at greater depths results in a change from a 4.5-fold difference in metabolic rate at the surface between the lowest and highest curve to a 1.8-fold difference at $1000 \mathrm{~m}$. The highly similar rates in the lower mesopelagic zone (500 to $1000 \mathrm{~m}$, Fig. 2), the similarity in ocean temperatures at those depths, and the fact that similar or identical species are ubiquitous at those depths suggest that metabolism of micronektonic Crustacea is essentially the same between 500 and $1000 \mathrm{~m}$ thoughout much of the world ocean.

Selective pressures determining the metabolic character of micronektonic Crustacea appear to operate most strongly in the well-illuminated upper layers of the water column. Data from the present study showing a metabolic decline in species from a nearly isothermal water column, and the comparison between systems discussed above, strongly support the contention that the metabolic decline observed in deeper-living Crustacea and fishes (Torres et al. 1979, Torres \& Somero 1988) is the result of a relaxation of selective pressures relating to visual predation near the surface (cf. Childress et al. 1990, Cowles et al. 1991). The absence of refugia in the pelagic environment dictates a need for a well developed swimming ability in species dwelling in or migrating to near surface waters. Deeper-living species may enjoy the luxury of energy-conserving lower metabolic rates in the darkness typical of the lower mesopelagic zone.

Species dwelling in the Antarctic system are afforded a seasonal relaxation in visual predation by the long nights, or, at higher latitudes, the $24 \mathrm{~h}$ darkness of the Antarctic winter. In a similar manner to deep-living mesopelagic species, the lowered winter metabolism observed in the present study in 3 epipelagic species is best accommodated in an environment where predation pressure is minimized by a cloak of darkness.

Acknowledgements. This paper is dedicated to Jose Torres (1920-1992), philosopher and scholar The research was supported by NSF DPP 8420652, DPP 8819533, and OPP 9220493 to J.J.T. and T.L.H., INT 8903589 and DPP 9000608 to J.J.T., and DPP 8419894 to D.G.A. We thank G. Somero and S. Kaupp for their time and expertise in teaching us the assays for LDH, CS, and RNA/DNA. We thank the captains and crews of the RV 'Melville', 'Polar Duke', and the USCGC 'Glacier' for their support. Kendra Daly has been an invaluable source of support both on board ship and during manuscript preparation. Many thanks to our other colleagues in the AMERIEZ program for sharing data and lending support at sea. 


\section{LITERATURE CITED}

Alldredge, A. L., Robison, B. H., Fleminger, A., Torres, J. J., King, J. M., Hamner, W. M. (1984). Direct sampling and in situ observation of a persistent copepod aggregation in the mesopelagic zone of the Santa Barbara Basin. Mar. Biol. 80: 75-81

Burggren, W., Roberts, J. L. (1991). Respiration and metabolism. In: Prosser, C. L. (ed.) Environmental and metabolic animal physiology. Wiley-Liss, New York, p. 353-436

Childress, J. J (1971). Respiratory rate and depth of occurrence of midwater animals. Limnol. Oceanogr. 16: 104-106

Childress, J. J. (1975). The respiratory rates of midwater crustaceans as a function of depth of occurrence and relation to the oxygen minimum layer off southern California. Comp. Biochem. Phyiol. 50A: 787-799

Childress, J. J., Barnes, A. T., Quetin, L. B., Robison, B. H. (1978). Thermally protecting cod-ends for recovery of living deep-sea animals. Deep Sea Res. 25: 419-422

Childress, J. J., Cowles, D. L., Favuzzi, J. A., Mickel, T J. (1990). Metabolic rates of benthic deep-sea decapod crustaceans decline with increasing depth primarily due to the decline in temperature. Deep Sea Res. 37: 929-949

Childress, J. J., Nygaard, M. H. (1973). The chemical composition of midwater fishes as a function of depth of occurrence off Southern California. Deep Sea Res. 20: 1093-1109

Childress, J. J., Nygaard, M. H. (1974). Chemical composition and buoyancy of midwater crustaceans as a function of depth of occurrence off southern California. Mar. Biol. 27 : $225-238$

Childress, J. J., Somero, G. N. (1979). Depth-related enzymic activities in muscle, brain, and heart of deep-living pelagic marine teleosts. Mar. Biol. 52: 273-283

Clark, L. C. (1956). Monitor and control of blood and tissue oxygen tensions. Trans. Am. Soc. Art. Int. Orgs. 2: 41-48

Cowles, D. L., Childress, J. J., Wells, M. E. (1991). Metabolic rates of midwater crustaceans as a function of depth of occurrence off the Hawaiian Islands: food availability as a selective factor? Mar. Biol. 110: 75-83

Donnelly, J., Torres, J. J. (1988). Oxygen consumption of midwater fishes and crustaceans from the eastern Gulf of Mexico. Mar. Biol. 97: 483-494

Donnelly, J., Torres, J. J., Hopkins, T. L., Lancraft, T. M. (1990). Proximate composition of Antarctic mesopelagic fishes. Mar. Biol. 106: 13-23

Everson, I. (1984). Marine zooplankton. In: Laws, R. M. (ed.) Antarctic ecology, Vol. 2. Academic Press, London, p. $463-490$

Gordon, A. L., Molinelli, E. J., Baker, T. N. (1982). Southern Ocean atlas. Columbia University Press, New York

Heusner, A. A. (1982). Energy metabolism and body size. I. Is the 0.75 mass exponent of Kleiber's equation a statistical artifact? Respir. Physiol. 48: 1-12

Heusner, A. A. (1987). What does the power function reveal about structure and function in animals of different size? A. Rev. Physiol. 49: 121-133

Hopkins, T. L., Gartner, J. V., Flock, M. E. (1989). The caridean shrimp (Decapoda: Natantia) assemblage in the mesopelagic zone of the eastern Gulf of Mexico. Bull. mar. Sci. 45: 1-14

Hopkins, T. L., Lancraft, T M., Torres, J. J., Donnelly, J. (1993). Community structure and trophic ecology of zooplankton in the Scotia Sea marginal ice zone in winter (1988). Deep Sea Res. 40: 81-105

Ikeda, T. (1981). Metabolic activity of larval stages of Antarctic krill. Antarct. J. U.S. 16(5): 161-162

Ikeda, T. (1988). Metabolism and chemical composition of crustaceans from the Antarctic mesopelagic zone. Deep Sea Res. 35: 1991-2002

Ikeda, T., Dixon, P. (1982). Body shrinkage as a possible overwintering mechanism of the Antarctic krill, Euphausia superba Dana. J. exp. mar. Biol. Ecol. 62: 143-151

Ikeda, T., Dixon, P. (1984). The influence of feeding on the metabolic activity of Antarctic krill (Euphausia superba Dana). Polar Biol. 3: 1-9

Iwasaki, N., Nemoto, T (1987). Distribution and community structure of pelagic shrimps in the Southern Ocean between $150^{\circ} \mathrm{E}$ and $115^{\circ} \mathrm{E}$. Polar Biol. 8: 121-128

Kawaguchi, K., Ishikawa, S., Matsuda, O. (1986). The overwintering strategy of Antarctic krill (Euphausia superba Dana) under the coastal fast ice off the Ongul Islands in Lutzow-Holm Bay, Antartica. Mem. natn. Inst. polar Res. (spec. iss.) $44: 67-85$

Lancraft, T. M., Hopkins, T. L., Torres, J. J., Donnelly, J. (1991). Oceanic micronektonic/macrozooplanktonic community structure and feeding in ice covered Antarctic waters during the winter. Polar Biol. 11: 157-167

Lancraft, T M., Torres, J. J., Hopkins, T. L. (1989). Micronekton and macrozooplankton in the open waters near Antarctic ice edge zones (AMERIEZ 1983 and 1986). Polar Biol. 9: 225-233

Madin, L. P., Harbison, G. R. (1977). The associations of Amphipoda Hyperiidae with gelatinous zooplankton. I. Associations with Salpidae. Deep Sea Res. 24: 449-463

Nagata, K. (1986). Amphipod crustaceans from surface waters of the Southern Ocean during 1983-1984 summer. Mem. natn. Inst. polar Res. (spec. iss.) 40: 259-276

Quetin, L. B., Ross, R. M. (1991). Behavioral and physiological characteristics of the Antarctic krill, Euphausia superba. Am. Zool. 31: 49-64

Quetin, L. B. Ross, R. M., Uchio, K. (1980). Metabolic characteristics of midwater zooplankton: ammonia excretion, $O: N$ ratios, and the effect of starvation. Mar. Biol. 59: 201-209

Scholander, P. F., Flagg, W., Walter, V., Irving, L. (1953). Climatic adaptation in arctic and tropical poikilotherms. Physiol. Zool. 26: 67-92

Smith, W. O., Nelson, D. M. (1990). Phytoplankton growth and new production in the Weddell Sea marginal ice zone during austral spring and autumn. Limnol. Oceanogr. 35: $809-821$

Smith, W. O., Sakshaug, E. (1990). Polar phytoplankton. In: Smith, W. O. (ed.) Polar oceanography. Academic Press, New York, p. 477-526

Snedecor, G. W., Cochran, W. G. (1967). Statistical methods. lowa State University Press, Ames

Somero, G. N. (1991). Hydrostatic pressure and adaptation to the deep sea. In: Prosser, C. L. (ed.) Environmental and metabolic animal physiology. Wiley-Liss, New York, p. 167-204

Torres, J. J., Belman, B. W., Childress, J. J. (1979). Oxygen consumption rates of midwater fishes as a function of depth of occurrence. Deep Sea Res. 26: 185-197

Torres, J. J., Donnelly, J., Hopkins, T. L., Lancraft, T M., Aarset, A. V., Ainley, D. G. (1994). Proximate compositon and overwintering strategies of Antarctic micronektonic Crustacea. Mar. Ecol. Prog. Ser. 113: 221-232

Torres, J J., Somero, G. N. (1988). Metabolism, enzymic activities, and cold adaptation in Antarctic mesopelagic fishes. Mar. Biol. 98: 169-180

Zwalley, H. H., Comiso, J. C., Parkinson, C. L., Campbell, W. J. Carsey, F. D., Gloerson, P. (1983). Antarctic sea ice. 1973-1976: satellite passive microwave observations. NASA spec. publ. 459. National Aeronautics and Space Administration, Washington, DC 